\title{
Validation of snoring detection using a smartphone app
}

\author{
Jui-Kun Chiang ${ }^{1} \cdot$ Yen-Chang Lin $^{2} \cdot$ Chih-Wen Lin ${ }^{3,4} \cdot$ Ching-Shiung Ting $^{5} \cdot$ Yi-Ying Chiang ${ }^{2} \cdot$ Yee-Hsin Kao ${ }^{6}$
}

Received: 17 July 2020 / Revised: 7 January 2021 / Accepted: 24 March 2021 / Published online: 3 April 2021

(C) The Author(s) 2021

\begin{abstract}
Purpose Snoring is closely related to obstructive sleep apnea in adults. The increasing abundance and availability of smartphone technology has facilitated the examination and monitoring of snoring at home through snoring apps. However, the accuracy of snoring detection by snoring apps is unclear. This study explored the snoring detection accuracy of Snore Clock—a paid snoring detection app for smartphones.

Methods Snoring rates were detected by smartphones that had been installed with the paid app Snore Clock. The app provides information on the following variables: sleep duration, snoring duration, snoring loudness (in $\mathrm{dB}$ ), maximum snoring loudness (in $\mathrm{dB}$ ), and snoring duration rate (\%). In brief, we first reviewed the snoring rates detected by Snore Clock; thereafter, an ear, nose, and throat specialist reviewed the actual snoring rates by using the playback of the app recordings.

Results In total, the 201 snoring records of 11 patients were analyzed. Snoring rates measured by Snore Clock and those measured manually were closely correlated $(r=0.907)$. The mean snoring detection accuracy rate of Snore Clock was 95\%, with a positive predictive value, negative predictive value, sensitivity, and specificity of $65 \% \pm 35 \%, 97 \% \pm 4 \%, 78 \% \pm 25 \%$, and $97 \% \pm 4 \%$, respectively. However, the higher the snoring rates, the higher were the false-negative rates for the app.

Conclusion Snore Clock is compatible with various brands of smartphones and has a high predictive value for snoring. Based on the strong correlation between Snore Clock and manual approaches for snoring detection, these findings have validated that Snore Clock has the capacity for at-home snoring detection.
\end{abstract}

Keywords Snoring $\cdot$ Snore Clock $\cdot$ Smartphones $\cdot$ App

Yee-Hsin Kao

m2200767@gmail.com

1 Department of Family Medicine, Dalin Tzu Chi Hospital, Buddhist Tzu Chi Medical Foundation, 2, Minsheng Road, Dalin 62247, Chiayi, Taiwan

2 Nature Dental Clinic, 341, Sec. 3, Zhongshan Rd, Puli Township, Nantou 545, Taiwan

3 Departments of School of Medicine, Tzu Chi University, Hualien, Taiwan

4 Department of Medical Imaging, Dalin Tzu Chi Hospital, Buddhist Tzu Chi Medical Foundation, Chiayi, Taiwan

5 Department of Otolaryngology, Tainan Municipal, 670 Chung-Te Road, Tainan 70173, Taiwan

6 Department of Family Medicine, Tainan Municipal Hospital (Managed by Show Chwan Medical Care Corporation), 670 Chung-Te Road, Tainan 70173, Taiwan

\section{Introduction}

Snoring, defined as "the vibration of palatal soft tissue from obstruction of air movement on breathing during sleeping," is believed to be a key indicator of possible obstructive sleep apnea syndrome (OSA) [1]. Snoring prevalence in Asian countries, in descending order, is Taiwan (59\%) [2], Malaysia (47\%) [3], Turkey (41\%) [4], Japan (24\% among men and $10 \%$ among women) [5], Singapore (7\%) [6], and Thailand (5\%) [7]. Snoring prevalence also differs among ethnicities. The higher snoring prevalence in Chinese descents compared with Caucasians might be due to Chinese people having narrower cranial bases and flatter mid-face structures [8].

OSA is closely associated with several serious illnesses, including systemic hypertension, coronary artery disease, stroke, and metabolic syndrome; it may also result in motor vehicle accidents and diminished quality of life [9-11]. OSA is insidious, and patients are often unaware of the associated symptoms. The cardinal manifestations of OSA include loud snoring, breathing pauses during sleep, fitful sleep quality, 
and excessive daytime sleepiness [12]. Although not all patients with snoring have clinically significant sleep apnea, snoring is the earliest and most common symptom of OSA, occurring in $70-95 \%$ of patients with OSA $[13,14]$. In addition, snoring intensity increases with OSA severity [14], and snoring itself can present a vascular risk [15].

One related study distinguished between two types of snore: the first type has a very low frequency $(<20 \mathrm{~Hz})$ but largeamplitude vibrations lasting from 0 to $1 \mathrm{~s}$ and 2.5 to $4.5 \mathrm{~s}$; and the second type involves high-frequency sounds with small-amplitude vibrations lasting from 1.0 to $1.5 \mathrm{~s}$ and 4.5 to $5.0 \mathrm{~s}$ [15]. Another study reported that snores can be categorized into irregular and regular snores and noted that patients with severe OSA had a shorter interval between regular snores [16]. Various obstruction sites also induce different snoring frequencies. Common obstruction sites include the velum, oropharynx, tongue base, and epiglottis [17]. The frequencies of more severe snoring differ considerably from those of habitual snoring, which range from 110 to $190 \mathrm{~Hz}[18,19]$. However, in patients with OSA, the snoring frequency might be higher than $800 \mathrm{~Hz}[20,21]$. Notably, the average snoring sound intensity is higher for men than for women [19]. Polysomnography (PSG) is the gold standard method for diagnosing OSA and monitoring snoring [13]. However, PSG must be performed at special sleep treatment centers where patients sleep overnight. Patients undergoing PSG may experience some inconveniences. First, many patients do not sleep well during PSG examination because of the discomfort resulting from the cumbersome monitoring wires required for this test. Second, patients might face wait times of several weeks or even longer for a PSG examination in Taiwan. Third, the PSG test is a time-consuming procedure that can result in considerable patient discomfort [22]. Therefore, simplified recording and monitoring instruments that can conveniently and reliably diagnose snoring and OSA at home are necessary [23]. A related study suggested that sleep physicians consider repeat testing with home sleep apnea tests (HSATs) in patients with a negative PSG and clinical symptoms of OSA [24]. HSAT devices are classified by the United States Food and Drug Administration (FDA) as Class II medical devices, which the FDA defines as "devices for which general controls are insufficient to provide reasonable assurance of the safety and effectiveness of the device" [25]. However, HSATs have some limitations. For example, mispositioned pulse oximeters can yield false low readings [26]. HSAT devices that include cardiorespiratory portable monitors have sensors that can detect breathing patterns and pulse oxygen saturation, masks with tubes for insertion into patients' nostrils, and sensors for patients' abdomen and chest. These devices may interrupt sleep and be inconvenient for most patients, who may be unable to afford the cost of using HSAT devices at home every night. Finally, raw data from HSAT devices cannot be read directly and must be reviewed and interpreted by a certified physician.
Along with advances in science and technology, the functions of smartphones have expanded dramatically and rapidly. In addition to their communication features, smartphones allow users to download apps through online stores. More than 100,000 health apps are available on the Android and iOS platforms, with one target for health and fitness apps being sleep and sleep hygiene [27]. Snoring apps are software applications that run on smartphones and record sound information while the user sleeps; such apps have been available for several years and enable convenient and personalized sleep care [28]. Snoring apps are undoubtedly a simple and feasible option for monitoring snoring. With the progress of innovative monitoring mechanisms and techniques, these apps can be used regularly at home for examinations without interrupting a person's sleep. The precision of smartphone apps for predicting snoring reportedly ranges from 93 to $96 \%$, with a sensitivity ranging from 64 to $96 \%$; however, app performance can vary greatly among smartphone models [29]. This study explored snoring detection accuracy in one of these smartphone apps, Snore Clock.

\section{Materials and methods}

\section{Patients and methods}

Patients with snoring problems using the Lin oral appliance (I602555 [Taiwan], ZL 201310753192.9 [China]) [30] were recruited from a dental clinic. The inclusion criteria were patients with snoring problems who volunteered to participate in this study. The patients independently downloaded the paid app SnoreClock. Patients aged $<20$ years were excluded from the study. All patients placed their smartphones within arm's reach just before falling asleep. Smartphones were positioned on the upper side of the shoulder within $30 \mathrm{~cm}$ of the head for optimal recording of snoring sounds. However, no special restrictions with respect to placing the phone on the bed or on a bedside table were applied. The patients recorded their snoring using SnoreClock, following their natural sleep habits for 2-3 weeks, after which the smartphones were returned to the researchers.

\section{Snore Clock}

Snore Clock provides the following data on the duration and intensity of snoring: sleep duration, snoring duration, snoring loudness $(\mathrm{dB})$, maximum snoring loudness $(\mathrm{dB})$, and rate of snoring duration (\%). Snore Clock also has a feature that enables users to focus on particular snoring events to facilitate playback of the most notable snoring sounds. This app is available for both iOS and Android devices. The author of Snore Clock was not involved in this study. 
All the recordings from Snore Clock were replayed and verified. Snoring was defined as snorting or grunting sounds while asleep. If Snore Clock displayed signals with no spiking waves, snoring was likely to be absent. However, segments of spiking waves indicated a higher possibility of snoring. The app marked snoring periods (including snoring signals and pauses) with red bands and calculated the rate of total snoring duration for all recording sessions.

Before we determined the presence of snoring signals, we conducted a test: the snoring of a randomly selected case (28year-old man; body mass index $=23.3$ ) was recorded simultaneously with a digital sound recorder using linear pulse-code modulation (ICD SX-2000, Sony Electronics Inc., Tokyo, Japan) and Snore Clock during sleep. The sound recorder had two built-in high-performance dynamic microphones. We set the low-cut filter switch to "OFF" in order to obtain snoring and breathing sounds, and we set the sampling rate to $44,100 \mathrm{~Hz}$. We analyzed the dominant frequencies every 0.01 s. We carefully listened to this recording on the computer and compared the results between the digital recorder and SnoreClock. We noted that interrupted sounds with a large volume (higher $\mathrm{dB}$ in the app) were easily recognized as snoring by the app. These noisy sounds included signals with low frequencies $(<1000 \mathrm{~Hz})$ or high frequencies (wide frequency bands, as high as $15,762 \mathrm{~Hz}$ ). However, sounds with smaller volumes with frequency spectrograms resembling snoring sounds on the computer were generally neglected by this app. These sounds were similar to stronger expiration air flows on the smartphone (frequencies $<5000 \mathrm{~Hz}$ ). All these processes required $>10 \mathrm{~h}$ to complete.

We then carefully listened to the recordings; any equivocal sounds were confirmed by an ear, nose, and throat (ENT) specialist (Ching-Shiung Ting, M.D.): this is referred to as the "manual method" hereafter. The snoring epoch is composed of several snoring signals interrupted by pauses $(<10$ s) [31]. Therefore, the duration of snoring was represented by the sum of total snoring epochs.

The study protocol was reviewed and approved by the Research Ethics Committee of the Buddhist Dalin Tzu Chi Hospital in Taiwan (No. B10703013).

\section{Statistical analysis}

Statistical software R (version 3.6.1) was used for all statistical analyses. Statistical significance was set at $p<0.05$, and all tests were two-tailed. Categorical variables are presented as frequency and percentage, and continuous variables are presented as mean \pm standard deviation. Univariate linear regression analyses and correlations were performed to evaluate the factors associated with snoring rates. The sensitivity, specificity, positive predictive value, and negative predictive value of the app were calculated. Analysis of variance (ANOVA) was performed to test the mean differences between groups.

\section{Results}

In total, 220 recordings from 11 patients were collected. We excluded recordings of $<5 \mathrm{~h}$ (19 recordings) and finally included 201 recordings for further analysis. Three Asus ZenFone Max Plus (M1), three Apple iPhone 6, three Sony Xperia, one HTC One, and one Xiaomi Redmi 5 were used for the recordings. Short recordings $(<5 \mathrm{~h})$ were excluded because they might be reflective of patients with poor sleep or insufficient phone battery power. The patients' basic demographic data are presented in Table 1 . No patient had a history of diabetes, respiratory disease, sleep disorders, or alcohol or smoking problems.

The mean accuracy rate for snoring detection by SnoreClock was $95 \%$ (comprising 9\% snoring rate and $86 \%$ non-snoring rate, approved both by the app and manual method). The mean snoring rates calculated by SnoreClock were only $0.3 \%$ less than those calculated using the manual method $(11.3 \% \pm 10.8 \%$ vs. $11.6 \% \pm 12.9 \%$; Table 2$)$. The snoring rates measured by SnoreClock were highly correlated with the snoring rates measured using the manual method $(r=0.907, \beta$ $=1.09, p<0.001$; Fig. 1$)$. Moreover, the false-negative rates for SnoreClock positively correlated with the false-negative rates for the manual method ( $r=0.431, \beta=1.06, p<0.001$; Table 3 and Fig. 2). The snoring rates determined using SnoreClock were then compared with those obtained using the manual method. The mean values of SnoreClock's sensitivity, specificity, negative predictive value, and positive predictive value were $78 \% \pm 25 \%, 97 \% \pm 4 \%, 97 \% \pm 4 \%$, and $65 \% \pm 35 \%$, respectively (Table 4 ). Sensitivity versus $1-$ specificity was plotted with a smoothing line (Fig. 3). Different points according to different sensitivities and specificities had distinct positive and negative predictive values. We carefully calculated the area under the receiver operating characteristic curve (AUC) for every recording (201 AUCs for 201 recordings). The mean value of the AUC was $0.946 \pm 0.528$. Thus, Snore Clock had a high predictive value for snoring.

These recordings were time-series data. Each night's sleep data were represented on $941 \pm 199$ px (range 540-1125 px) on the smartphone screens. Therefore, 1 px represented $28.0 \pm$ $7.8 \mathrm{~s}$. The app displayed the time bands of snoring on the screen. Manual snoring bands could also be drawn. We compared these snoring bands for every record. The chance of making a type II error (i.e., false-negative rate) is known as
Table 1 Basic demographic characteristics of subjects

\begin{tabular}{ll}
\hline Variables & $n=11$ \\
\hline Gender (male/female) & $7 / 4$ \\
Age, years & $38.2 \pm 11.9$ \\
Body mass index & $23.6 \pm 3.2$ \\
Hypertension history, yes & 1 \\
\hline
\end{tabular}


Table 2 Snoring rates estimated by SnoreClock app and manual method

\begin{tabular}{ll}
\hline Variables & Results \\
\hline Records enrolled for analysis & 201 \\
Records time, hours & $7.0 \pm 1.1$ \\
Snoring rates by SnoreClock app, \% & $11.3 \pm 10.8$ \\
Snoring rates by manual method, \% & $11.6 \pm 12.9$ \\
Difference of snoring rates by app-manual method, \% & $-0.3 \pm 5.5$ \\
Absolute difference of snoring rates between app and manual method, $\%$ & $3.6 \pm 4.2$ \\
No snoring, but app determined snoring, rate, $\%$ & $2.6 \pm 3.2$ \\
Snoring yes, but app determined no snoring, rate, $\%$ & $2.8 \pm 4.4$ \\
Snoring rates, both by app and manual method, \% & $8.8 \pm 10.3$ \\
No snoring, both by app and manual method, $\%$ & $85.8 \pm 13.3$ \\
\hline
\end{tabular}

The results were shown as mean $\pm \mathrm{SD}$ beta $(\beta)$. We examined the false-negative rate for every recording. The power, represented by $1-\beta$, was acquired for every recording, and the mean power in our study was $97 \% \pm$ $4 \%$. The mean differences in snoring rates between the measurements by SnoreClock and estimation from the manual method for different phone brands were within $\pm 5 \%$. The $p$ value of ANOVA for these mean differences between the iOS group and non-iOS group was 0.802 .

\section{Discussion}

In the current study, we used the variable snoring rate (i.e., snoring duration or sleep duration) to determine Snore Clock's accuracy. A strong correlation was observed between the results obtained through a manual measurement of snoring and Snore Clock, which were reviewed by an ENT specialist. The correlation between SnoreClock and the manual method for detecting snoring was $0.907(p<0.001)$.

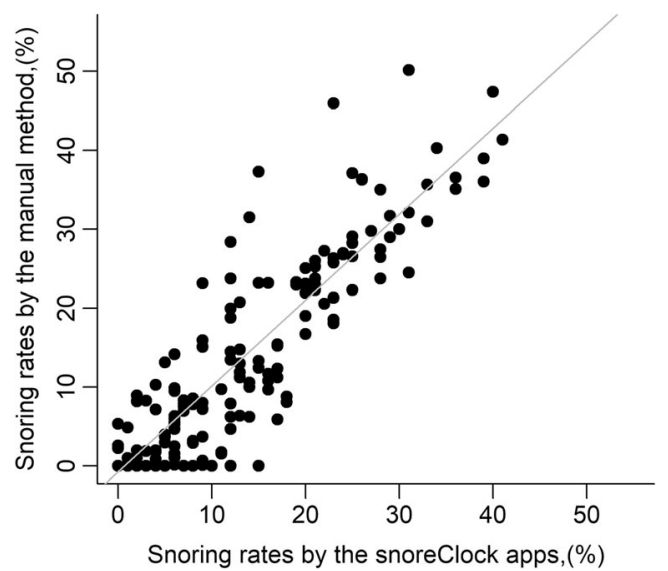

Fig. 1 Scatter plot of snoring rates determined by Snore Clock and a manual method of measurement
The results indicated that SnoreClock is a highly accurate app for detecting snoring. The findings also revealed that the snoring rate was significantly underestimated if SnoreClock indicated a higher snoring rate.

The positive predictive value for snoring was $65 \% \pm$ $35 \%$ in the current study; elsewhere, the results have been $70 \%$ [22] and 93-96\% [29]. In Shin's study [22], researchers detected snoring using a smartphone app at users' homes. Non-snore noises are more common at home, which affected both the present and Shin's study; such noises were less of a concern in Camacho's study [29], which was conducted in a hospital. Non-snore noises in patients' sleep areas, such as bedding noise, coughing, and environmental noise [32], might be the causes of false-positive snoring recorded in this study. In addition, in their study, Camacho used the app Quit Snoring and compared the results with in-laboratory PSG data. However, they used data from only two patients

Table 3 The associations between the variables listed and the snoring rates by SnoreClock app

\begin{tabular}{|c|c|c|c|}
\hline Variables & Correlation & $\beta$ & $\begin{array}{l}p \\
\text { value }\end{array}$ \\
\hline Record time, hour & -0.021 & -0.22 & 0.763 \\
\hline Snoring rate by manual method, $\%$ & 0.907 & 0.76 & $<0.001$ \\
\hline $\begin{array}{l}\text { Difference of snoring rates by app-manual } \\
\text { method, } \%\end{array}$ & -0.172 & -0.34 & 0.015 \\
\hline $\begin{array}{l}\text { Absolute difference of snoring rates } \\
\text { between app and manual method, \% }\end{array}$ & 0.302 & 0.78 & $<0.001$ \\
\hline $\begin{array}{l}\text { Snoring rates, both by app and manual } \\
\text { method, \% }\end{array}$ & 0.956 & 1.00 & $<0.001$ \\
\hline $\begin{array}{l}\text { No snoring, both by app and manual } \\
\text { method, } \%\end{array}$ & -0.955 & -0.78 & $<0.001$ \\
\hline $\begin{array}{l}\text { No snoring, but app determined snoring, } \\
\text { rate, } \%\end{array}$ & 0.284 & 0.96 & $<0.001$ \\
\hline $\begin{array}{l}\text { Snoring yes, but app determined no } \\
\text { snoring, rate, } \%\end{array}$ & 0.431 & 1.06 & $<0.001$ \\
\hline
\end{tabular}




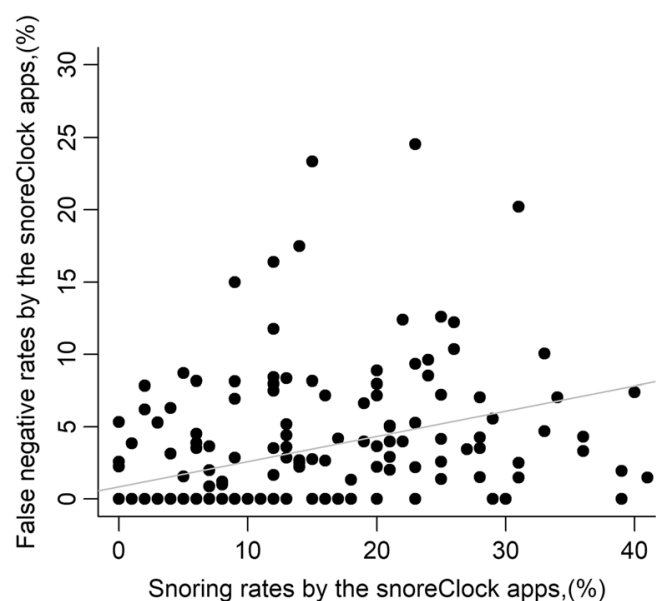

Fig. 2 Scatter plot of the false-negative snoring rates by Snore Clock vs. the rates by Snore Clock

undergoing in-laboratory PSG and one case study and set $53 \mathrm{~dB}$ as the snoring threshold in Quit Snoring. In the current study, we used snoring rate during sleep time as a variable for analysis because the snoring event is actually a period.

Another novel finding of the current study is that snoring rate might be underestimated if the snoring rate percentage is higher in SnoreClock. A similar result was also obtained in the study of Kreivi, where researchers recorded snoring with an MP3 recorder [33]. One reason for this might be that the sensitivity was set to a higher level to increase snoring detection [27]. Another reason might be the different frequencies of snoring to which the app was set.

\section{Limitations}

This study has limitations. First, snoring apps might be unable to distinguish between the snores of different people in a room. Second, the accuracy of Snore Clock was validated by an ENT specialist, not through PSG testing, despite PSG being the gold

Table 4 The mean parameters of snoring detection by SnoreClock app

\begin{tabular}{ll}
\hline Variables & Values \\
\hline Sensitivity, \% & $78.2 \pm 24.5$ \\
Specificity, \% & $97.0 \pm 3.6$ \\
False negative, \% & $21.8 \pm 24.5$ \\
False positive, \% & $3.0 \pm 3.6$ \\
Negative predictive value, \% & $97.0 \pm 3.6$ \\
Positive predictive value, \% & $65.3 \pm 35.2$ \\
AUC $*$, \% & $94.6 \pm 52.8$ \\
\hline
\end{tabular}

*AUC, area under the curve (receiver operating characteristic curve)

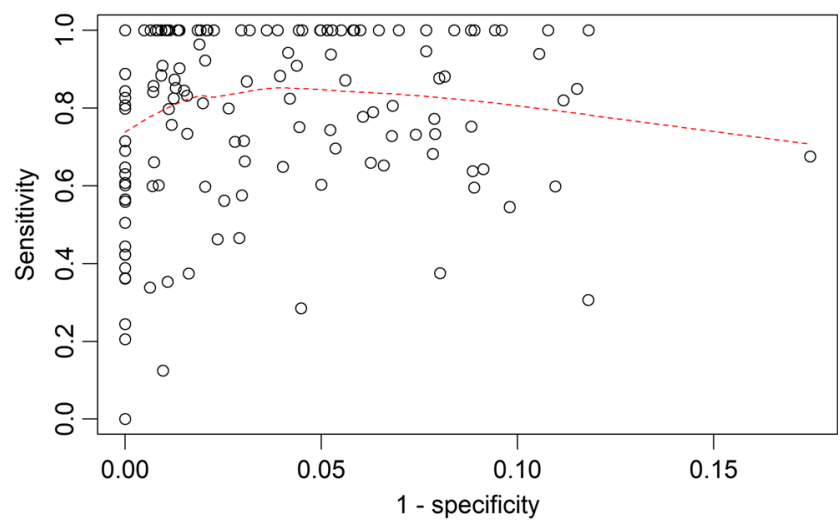

Fig. 3 Scatter plots of sensitivities versus 1 - specificities and the smoothing line

standard method to diagnose snoring. Third, the app might be unable to determine snoring when the sounds have low volumes or their frequencies are similar to those of the background. Fourth, the recording time represented sleep duration. The definite timing of falling sleep and waking up could not be detected by Snore Clock. Fifth, we experienced difficulty in investigating the hardware with regard to the bandwidth, amplification, and filter settings. These settings may have influenced the snoring detection output. Finally, all the patients in the present study were middle aged ( $30-52$ years), implying that the results cannot be extrapolated to other age groups.

\section{Conclusion}

The mean accuracy rate for snoring detection by SnoreClock was 95\%. The correlation between SnoreClock and the manual method for detecting snoring was strong. SnoreClock can be used on various smartphone brands and has a high predictive value for snoring. Our results validate at-home snoring detection through SnoreClock.

Abbreviations App, application;; AUC, area under the curve (receiver operating characteristic curve);; BMI, body mass index;; dB, decibel;; ENT, ear, nose, and throat;; HSATs, home sleep apnea tests;; Hz, hertz;; OSA, obstructive sleep apnea; PSG, polysomnography

Author's contributions YHK, CWL, and JKC contributed to conception, design, and drafting of the manuscript. JKC analyzed and interpreted the data. YCL, YCY, CST, and YYC wrote the manuscript. All authors revised and approved the final version. YH Kao and $\mathrm{CW}$ Lin contributed equally.

Funding JK Chiang received grants from the Dalin Tzu Chi Hospital, Buddhist Tzu Chi Medical Foundation (DTCRD108(2)-E-09). The funders had no role in study design, data collection and analysis, decision to publish, or preparation of the manuscript. The author of the SnoreClock app did not have involvement of this study. 


\section{Declarations}

Ethical approval The study protocol was reviewed and approved by the Research Ethics Committee of the Buddhist Dalin Tzu Chi Hospital in Taiwan (No. B10703013)

Informed consent Informed consent was obtained from all individual participants included in this study.

Conflict of interest The authors declare no competing interests.

Open Access This article is licensed under a Creative Commons Attribution 4.0 International License, which permits use, sharing, adaptation, distribution and reproduction in any medium or format, as long as you give appropriate credit to the original author(s) and the source, provide a link to the Creative Commons licence, and indicate if changes were made. The images or other third party material in this article are included in the article's Creative Commons licence, unless indicated otherwise in a credit line to the material. If material is not included in the article's Creative Commons licence and your intended use is not permitted by statutory regulation or exceeds the permitted use, you will need to obtain permission directly from the copyright holder. To view a copy of this licence, visit http://creativecommons.org/licenses/by/4.0/.

\section{References}

1. Nakano H, Hirayama K, Sadamitsu Y, Toshimitsu A, Fujita H, Shin S, Tanigawa T (2014) Monitoring sound to quantify snoring and sleep apnea severity using a smartphone: proof of concept. J Clin Sleep Med 10(1):73-78

2. Chuang LP, Hsu SC, Lin SW, Ko WS, Chen NH, Tsai YH (2008) Prevalence of snoring and witnessed apnea in Taiwanese adults. Chang Gung Med J 31(2):175-181

3. Kamil MA, Teng CL, Hassan SA (2007) Snoring and breathing pauses during sleep in the Malaysian population. Respirology 12(3):375-380

4. Kart L, Dutkun Y, Altin R, Ornek T, Kiran S (2010) Prevalence of major obstructive sleep apnea syndrome symptoms in coal miners and healthy adults. Tuberk Toraks 58(3):261-267

5. Nagayoshi M, Yamagishi K, Tanigawa T et al (2011) Risk factors for snoring among Japanese men and women: a community-based cross-sectional study. Sleep Breath 15(1):63-69

6. Khoo SM, Tan WC, Ng TP, Ho CH (2004) Risk factors associated with habitual snoring and sleep-disordered breathing in a multiethnic Asian population: a population-based study. Respir Med 98(6):557-566

7. Suwanprathes P, Won C, Komoltri C, Nana A, Kotchabhakdi N, Guilleminault C (2010) Epidemiology of sleep-related complaints associated with sleep-disordered breathing in Bangkok, Thailand. Sleep Med 11(10):1025-1030

8. Lam B, Ip MS, Tench E, Ryan CF (2005) Craniofacial profile in Asian and white subjects with obstructive sleep apnoea. Thorax 60(6):504-510

9. Yaggi HK, Concato J, Kernan WN, Lichtman JH, Brass LM, Mohsenin V (2005) Obstructive sleep apnea as a risk factor for stroke and death. N Engl J Med 353(19):2034-2041

10. Nieto FJ, Young TB, Lind BK, Shahar E, Samet JM, Redline S, D'Agostino RB, Newman AB, Lebowitz MD, Pickering TG (2000) Association of sleep-disordered breathing, sleep apnea, and hypertension in a large community-based study. Sleep Heart Health Study. JAMA 283(14):1829-1836

11. Young T, Peppard PE, Gottlieb DJ (2002) Epidemiology of obstructive sleep apnea: a population health perspective. Am J Respir Crit Care Med 165(9):1217-1239

12. Punjabi NM (2008) The epidemiology of adult obstructive sleep apnea. Proc Am Thorac Soc 5(2):136-143

13. Abeyratne UR, Wakwella AS, Hukins C (2005) Pitch jump probability measures for the analysis of snoring sounds in apnea. Physiol Meas 26(5):779-798

14. Maimon N, Hanly PJ (2010) Does snoring intensity correlate with the severity of obstructive sleep apnea? J Clin Sleep Med 6(5):475-478

15. Lee GS, Lee LA, Wang CY, Chen NH, Fang TJ, Huang CG, Cheng WN, Li HY (2016) The Frequency and Energy of Snoring Sounds Are Associated with Common Carotid Artery Intima-Media Thickness in Obstructive Sleep Apnea Patients. Sci Rep 6:30559

16. Mesquita J, Sola-Soler J, Fiz JA, Morera J, Jané R (2012) All night analysis of time interval between snores in subjects with sleep apnea hypopnea syndrome. Med Biol Eng Comput 50(4):373-381

17. Lee LA, Lo YL, Yu JF, Lee GS, Ni YL, Chen NH, Fang TJ, Huang CG, Cheng WN, Li HY (2016) Snoring Sounds Predict Obstruction Sites and Surgical Response in Patients with Obstructive Sleep Apnea Hypopnea Syndrome. Sci Rep 6:30629

18. Cavusoglu M, Kamasak M, Erogul O, Ciloglu T, Serinagaoglu Y, Akcam T (2007) An efficient method for snore/nonsnore classification of sleep sounds. Physiol Meas 28(8):841-853

19. Pevernagie D, Aarts RM, De Meyer M (2010) The acoustics of snoring. Sleep Med Rev 14(2):131-144

20. Perez-Padilla JR, Slawinski E, Difrancesco LM, Feige RR, Remmers JE, Whitelaw WA (1993) Characteristics of the snoring noise in patients with and without occlusive sleep apnea. Am Rev Respir Dis 147(3):635-644

21. Fiz JA, Abad J, Jane R et al (1996) Acoustic analysis of snoring sound in patients with simple snoring and obstructive sleep apnoea. Eur Respir J 9(11):2365-2370

22. Shin H, Cho J (2014) Unconstrained snoring detection using a smartphone during ordinary sleep. Biomed Eng Online 13:116

23. Dalmasso F, Benedetto G, Pogolotti R, Righini G, Spagnolo R (1990) Digital processing of snoring sounds. Eur Respir J Suppl $11: 528 \mathrm{~s}-532 \mathrm{~s}$

24. Lipatov K, Hayek A, Ghamande S, Boethel C, Chen W, Jones S (2018) Predictors of Obstructive Sleep Apnea on a Home Sleep Apnea Test After a Negative Attended Polysomnography. J Clin Sleep Med 14(11):1889-1894

25. U.S. FOOD \& DRUG ADMINISTRATION. $2020 \mathrm{https} / / / \mathrm{www}$. accessdata.fda.gov/scripts/cdrh/cfdocs/cfpcd/classification.cfm. Accessed 19 Dec 2020

26. Kapoor M (2019) Positive Home Sleep Apnea Test After a Negative Polysomnogram: Role of Potential Confounding Factors. J Clin Sleep Med 15(3):519-520

27. Ong AA, Gillespie MB (2016) Overview of smartphone applications for sleep analysis. World J Otorhinolaryngol Head Neck Surg 2(1):45-49

28. Shin H, Choi B, Kim D, Cho J (2014) Robust sleep quality quantification method for a personal handheld device. Telemed $\mathrm{J}$ E Health 20(6):522-530

29. Camacho M, Robertson M, Abdullatif J, Certal V, Kram YA, Ruoff CM, Brietzke SE, Capasso R (2015) Smartphone apps for snoring. J Laryngol Otol 129(10):974-979

30. Nature Dental Clinic. 2019 http://www.alliswell.tw/braces/ Accessed September 7, 2019 
31. Lee SA, Amis TC, Byth K, Larcos G, Kairaitis K, Robinson TD, Wheatley JR (2008) Heavy snoring as a cause of carotid artery atherosclerosis. Sleep 31(9):1207-1213

32. Dafna E, Tarasiuk A, Zigel Y (2013) Automatic detection of whole night snoring events using non-contact microphone. PLoS One 8(12):e84139
33. Kreivi HR, Salmi T, Maasilta P, Bachour A (2013) Screening of snoring with an MP3 recorder. Sleep Breath 17(1):77-84

Publisher's note Springer Nature remains neutral with regard to jurisdictional claims in published maps and institutional affiliations. 